\title{
Research Article \\ Etch Defect Characterization and Reduction in Hard-Mask-Based Al Interconnect Etching
}

\author{
Hong-Ji Lee, Che-Lun Hung, Chia-Hao Leng, Nan-Tzu Lian, Ling-Wu Young, \\ Tahone Yang, Kuang-Chao Chen, and Chih-Yuan Lu
}

Advanced Module Process Development Division, Technology Development Center, Macronix International Company, Ltd., (MD420) No.16, Li-Hsin Road, Science-Based Industrial Park, Hsinchu 300, Taiwan

Correspondence should be addressed to Hong-Ji Lee, jameslee@mxic.com.tw

Received 23 June 2008; Accepted 26 August 2008

Recommended by Jong-Shinn Wu

This paper identifies the defect adders, for example, post hard-mask etch residue, post metal etch residue, and blocked etch metal island and investigates the removal characteristics of these defects within the oxide-masked $\mathrm{Al}$ etching process sequence. Post hard-mask etch residue containing $\mathrm{C}$ atom is related to the hardening of photoresist after the conventional post-RIE ashing at $275^{\circ} \mathrm{C}$. An in situ $\mathrm{O}_{2}$-based plasma ashing on RIE etcher was developed to prevent the photoresist hardening from the high-ashing temperature; followed wet stripping could successfully eliminate such hardened polymeric residue. Post metal etch residue was caused from the attack of the $\mathrm{Al}$ sidewall by $\mathrm{Cl}$ atoms, and too much $\mathrm{CHF}_{3}$ addition in the $\mathrm{Al}$ main etch step passivated the surface of $\mathrm{Al}$ resulting in poor capability to remove the Al-containing residue. The lower addition of $\mathrm{CHF}_{3}$ in the $\mathrm{Al}$ main etch step would benefit from the residue removal. One possibility of blocked etch metal island creating was due to the micromasking formed on the opening of TiN during the hard-mask patterning. We report that an additional TiN surface pretreatment with the $\mathrm{Ar} / \mathrm{CHF}_{3} / \mathrm{N}_{2}$ plasmas could reduce the impact of the micromasking residues on blocked metal etch.

Copyright (C) 2008 Hong-Ji Lee et al. This is an open access article distributed under the Creative Commons Attribution License, which permits unrestricted use, distribution, and reproduction in any medium, provided the original work is properly cited.

\section{INTRODUCTION}

As the dimension of devices continues to scale down, the $\mathrm{Al}$ patterning by dry etching becomes challenging due to insufficient amount of photoresist to leave for etch rate fluctuation [1]. A damascene process is the highly potential approach to avoid the metal dry etching problems [2]. However, it is known that switching $\mathrm{Al}$ to $\mathrm{W}$ or $\mathrm{Cu}$ involvesadditional investments, both in terms of equipments and process development efforts [3]. Since Gabriel et al. [4] and Stojakovic and Ning [5] adopted hard mask to pattern $\mathrm{Al}$ in the dry etching process, it is worth exploring $\mathrm{Al}$ dry etching with hard mask in our $75 \mathrm{~nm}$ nonvolatile memory technology and beyond. With developing the TEOS hardmask-based $\mathrm{Al}$ patterning in our study, the etch defectivity is regarded as leading edge challenge in the manufacturing line. As illustrated in Figure 1, the defect types, referred to as "post hard-mask etch residue," "post metal etch residue," "blocked etch metal island," "particle," "bridging," "particulate bridging," and "corrosion," were found at the early stage of development. Interestingly, the major adders of post hard-mask etch residue, post metal etch residue, and blocked etch metal island were particularly high in the oxidemasked $\mathrm{Al}$ patterning but few in the conventional resistmasked $\mathrm{Al}$ patterning. From the observations of scanning electron micrographs as shown in Figure 1, these three defect types pose a tangible and substantial yield risk due to their subtle physical characteristics and high density on wafer. Hence, it is important to understand the behavior of these defect adders within the $\mathrm{Al}$ etching process sequence.

This paper describes the identification of the foregoing defect adders. Among these defect adders, post hard-mask etch residue is commonly observed after the oxide hardmask patterning followed by resist ashing and wet stripping. Such defect formation is found strongly related to the ashing process temperature. Post metal etch residue is found to be concentrated near the semi-iso metal sidewall damage and does not appear to be randomly distributed; the defect is demonstrated to be the attack of the metal sidewall by $\mathrm{Cl}$ atoms. One possibility of blocked etch metal island creating is the fine micromasking formed on the opening during the hard-mask patterning; the micromasking would cause 


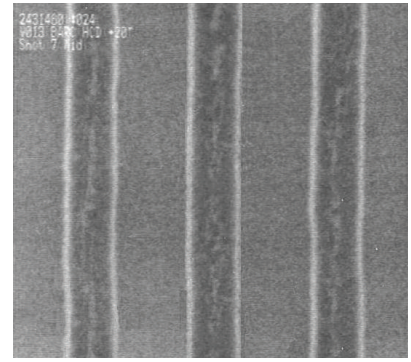

Post hard-mask etch residue (top of TEOS)

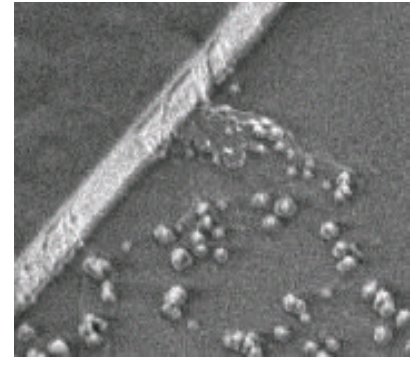

Post metal etch residue

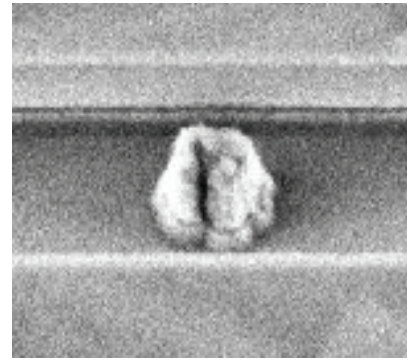

Blocked etch metal island (a)

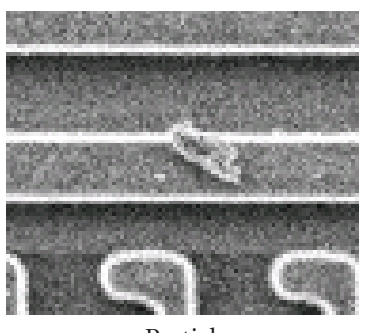

Particle

(d)

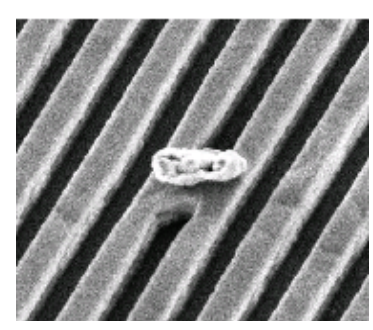

Bridging

(e)

(b)

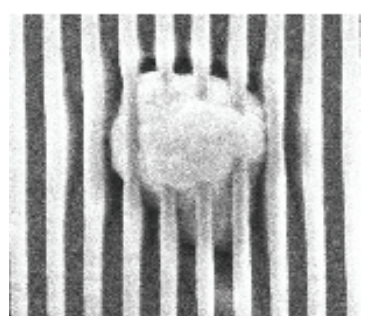

Particulate bridging

(f) (c)

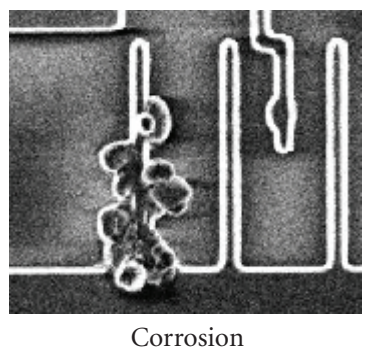

(g)

FIGURE 1: Examples of defect adders within the oxide-masked $\mathrm{Al}$ etching process sequence.

blocked metal etch resulting in metal island during the $\mathrm{Al}$ etching. The reduction of these defect adders optimizing $\mathrm{O}_{2}$ plasma ashing sequence, moderating the addition of $\mathrm{CHF}_{3}$ gas in the Al etching, and using additional TiN surface pretreatment prior to the TiN/Ti breakthrough step was reported.

\section{EXPERIMENT}

A blanket metal stack structure used in this study was deposited as follows (from bottom): PEOX/Ti/TiN/Al-0.5\% $\mathrm{Cu} / \mathrm{Ti} / \mathrm{TiN} / \mathrm{TEOS}$. Then, an organic antireflective film was coated on top of TEOS. The line patterns printed by using an ASML PAS-5500/700C DUV scanner with $420 \mathrm{~nm}$ resist. The hard-mask etching was carried out on an AMAT $\mathrm{MxP}^{+}$capacitively-coupled plasma dielectric etch system (see Figure 2(a)) with $\mathrm{CF}_{4} / \mathrm{CHF}_{3} / \mathrm{Ar} / \mathrm{N}_{2}$ chemistry followed by oxygen plasma ashing (SA-2000, PSC) at $275^{\circ} \mathrm{C}$ for 20 seconds and EKC265 wet stripping. EKC265 (EKC Technology Inc.) is a semiaqueous organic mixture formulated to remove resist residue generated after etch process. After the oxide hard mask was patterned, the remaining metal stack was etched on a Lam Research Alliance 9600PTX low-pressure, high-density, inductively coupledplasma metal etch system (see Figure 2(b)). The starting metal etch baseline consisted of three substeps, for example, the TiN/Ti breakthrough (BT) step with $\mathrm{Cl}_{2} / \mathrm{BCl}_{3} / \mathrm{CHF}_{3} / \mathrm{N}_{2}$ gas ratio $(10 / 2 / 3 / 2)$, the $\mathrm{Al}$ main etch (ME) step with $\mathrm{Cl}_{2} / \mathrm{BCl}_{3} / \mathrm{CHF}_{3} / \mathrm{N}_{2}$ gas ratio $(12 / 10 / 3 / 2)$, and the over-etch (OE) step with $\mathrm{Cl}_{2} / \mathrm{BCl}_{3} / \mathrm{CHF}_{3} / \mathrm{N}_{2}$ gas ratio $(12 / 14 / 3 / 2)$. The defect adders on wafer were inspected with a KLA-Tencor 2351 pattern inspection tool and a JW-7555S scanning electron microscopy (SEM). The chemical composition of the defect adder was analyzed using energy dispersive X-ray spectroscopy (EDS).

\section{RESULTS AND DISCUSSION}

\subsection{Characterization and reduction of post hard-mask etch residue}

A typical TEOS dielectric as hard mask was etched on $\mathrm{MxP}^{+}$with the following conditions: pressure $(200 \mathrm{mt})$; power $(600 \mathrm{~W}) \mathrm{CF}_{4} / \mathrm{CHF}_{3} / \mathrm{Ar} / \mathrm{N}_{2}$ gas ratio $(4 / 2 / 15 / 2)$, and $40 \%$ OE after the TEOS endpoint wasdetected. Figure 3 shows the SEM micrograph of TEOS that determines the sequent $\mathrm{Al}$ interconnect dimension, interestingly, keeping the backbone residues on top of the hard-mask structure. The residue was identified mainly containing $\mathrm{C}$ atom from the observation of its EDS spectrum (see Figure 3(c)). The data support the contention that the residue is related to the hardening of photoresist during the oxygen plasma ashing process; followed wet stripping is not effective in removing such hardened polymeric residue. According to the report of Gillespie et al. [6], it is helpful to reduce the hardened photoresist by lowering the ashing temperature. In this respect, we proposed an in situ $\mathrm{O}_{2}$-based plasma ashing process on $\mathrm{MxP}^{+}$etcher (cathode temperature: $15^{\circ} \mathrm{C}$ ) to prevent the photoresist hardening from the conventional post-RIE ashing at $275^{\circ} \mathrm{C}$. As seen in Figure 4, post hardmask etch residue on top of TEOS could be eliminated by optimizing in situ $\mathrm{O}_{2}$-based plasma ashing with rf power of $150 \mathrm{~W}$ for 12 seconds followed by EKC265 wet stripping. 


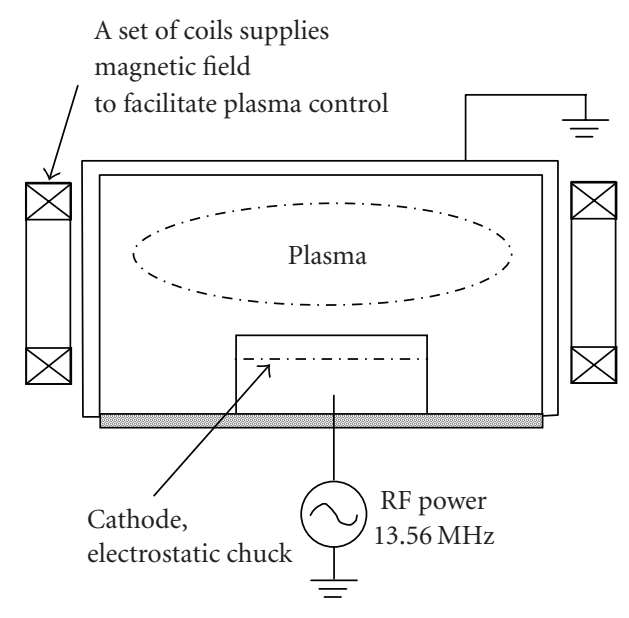

(a) Magnetically enhanced reactive ion etcher (MERIE)

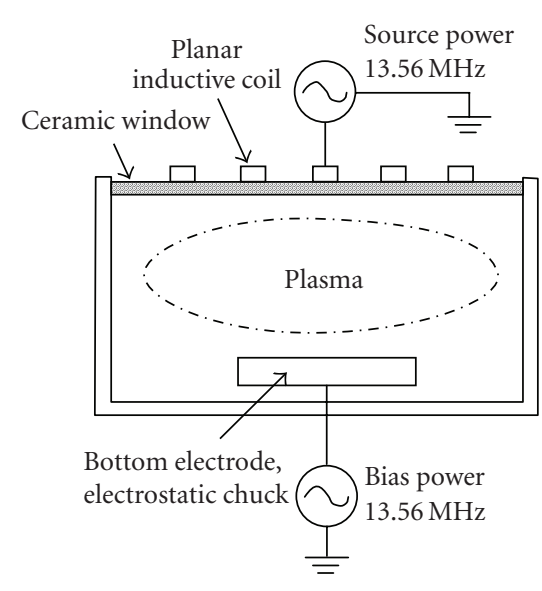

(b) ICP etcher

FIgURE 2: Schematic diagrams of etchers used in this study.

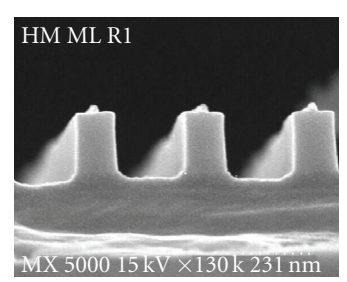

(a)

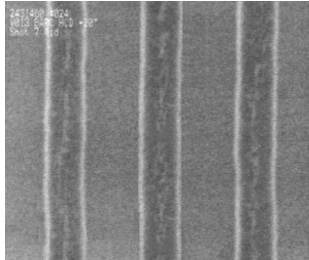

(b)

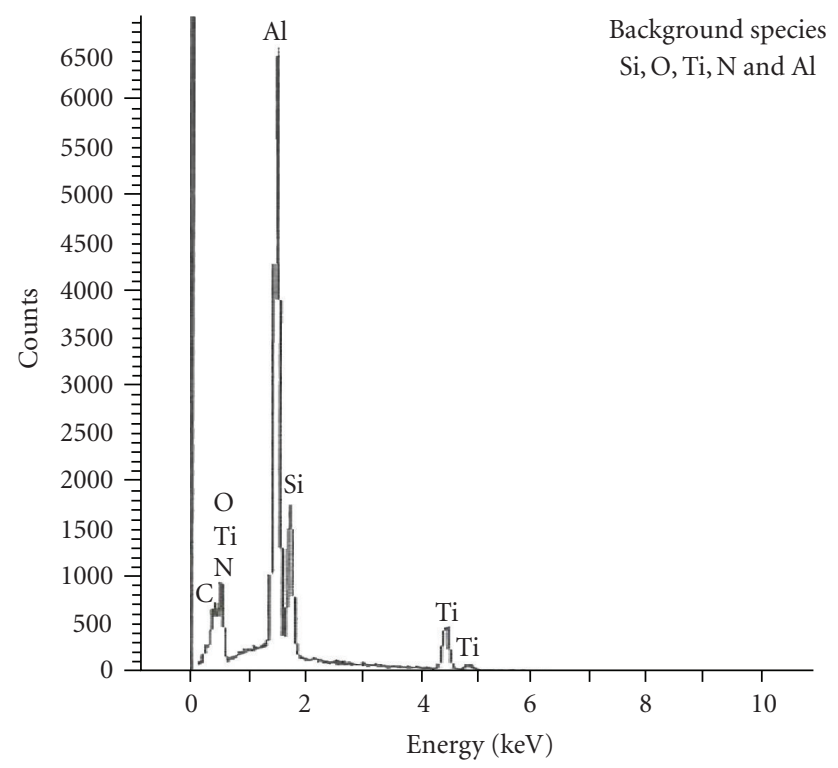

(c)

FIGURE 3: (a), (b) SEM micrographs of TEOS after etching. The backbone residues were found on top of TEOS, containing C atom identified by its EDS spectrum (c).

\subsection{Characterization and reduction of post metal etch residue}

After the TEOS hard mask was patterned, the remaining metal stack was etched on Alliance 9600PTX. Figure 5(a) shows the cross-sectional SEM micrograph of Al interconnect etched using high $\mathrm{CHF}_{3}(15 \mathrm{sccm})$ flow rate during the ME step in the starting etch baseline; the $\mathrm{Al}$ profile was tapered and notch-free. Subsequently, the defect inspection in die-to-die mode revealed the defect adder density around 


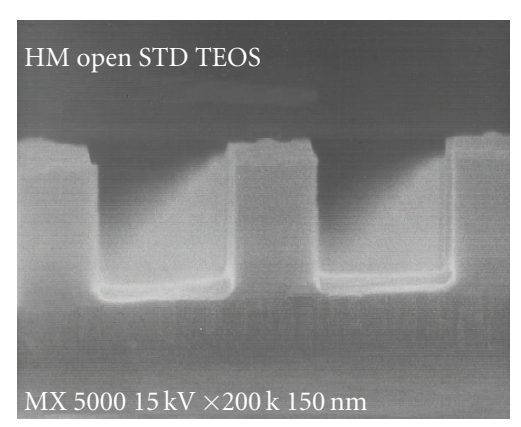

(a)

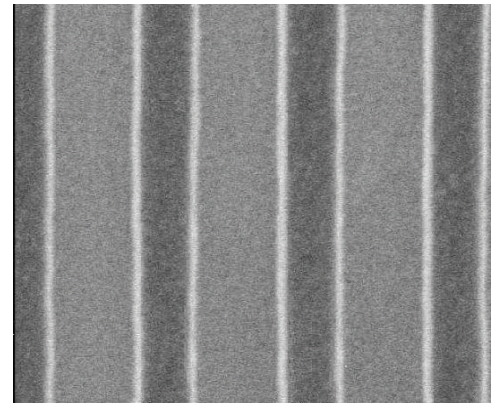

(b)

FIGURE 4: (a), (b) SEM micrographs of TEOS after etching with in situ $\mathrm{O}_{2}$-based plasma ashing followed by EKC265 wet stripping.

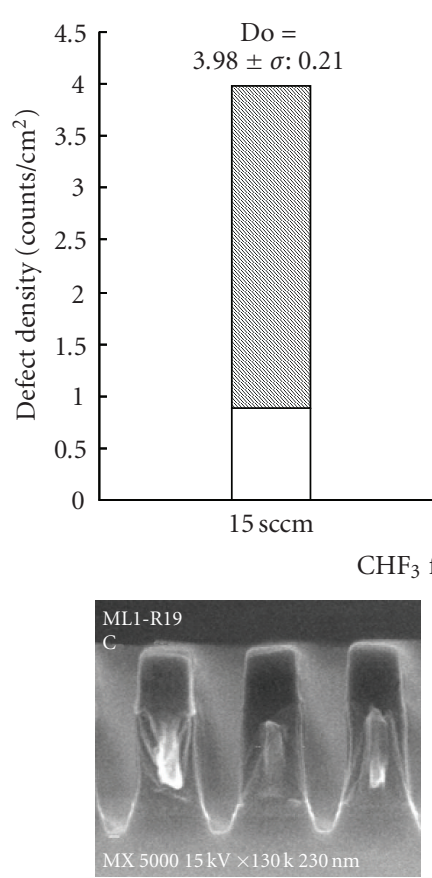

(a) $\mathrm{CHF}_{3}=15 \mathrm{sccm}$

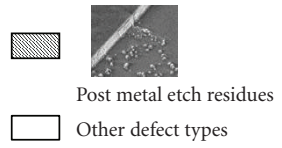

Do $=$

$1 \pm \sigma: 0.18$

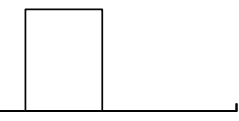

$6 \mathrm{sccm}$

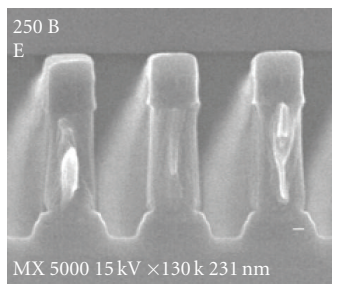

(b) $\mathrm{CHF}_{3}=6 \mathrm{sccm}$
FIGURE 5: (a) SEM micrograph of Al profile etched using $15 \mathrm{sccm}$ of $\mathrm{CHF}_{3}$ during the ME step. The major defect type is post metal etch residue ( $78 \%$ of total defect adders). (b) The ratio of post metal etch residue to total defect adders was decreased to $2.7 \%$ at the lower $\mathrm{CHF}_{3}$ flow of $6 \mathrm{sccm}$; however, there is a slight $\mathrm{Al}$ bottom notching observed from physical profile. Defect density (Do) was expressed as means \pm standard deviation $(\sigma)$ in counts $/ \mathrm{cm}^{2}$. Each etch condition was tested on three wafers.

3.98 defects $/ \mathrm{cm}^{2}$ in average, in which the major defect type is post metal etch residue (78\% of total defect adders). Upon SEM review, the residues are found to be concentrated near the semi-iso Al-damaged sidewall and do not appear to be randomly distributed. From the analysis of EDS spectrum (see Figure 6) attained from a representative residue adder, the defect contains $\mathrm{Al}, \mathrm{F}, \mathrm{Cu}, \mathrm{Si}$, and $\mathrm{Cl}$ atoms. The data indicate that the residues are related to the attack of the $\mathrm{Al}(0.5 \% \mathrm{Cu})$ sidewall by $\mathrm{Cl}$ atoms, and too much $\mathrm{CHF}_{3}$ addition in the ME step could passivate the surface of $\mathrm{Al}$

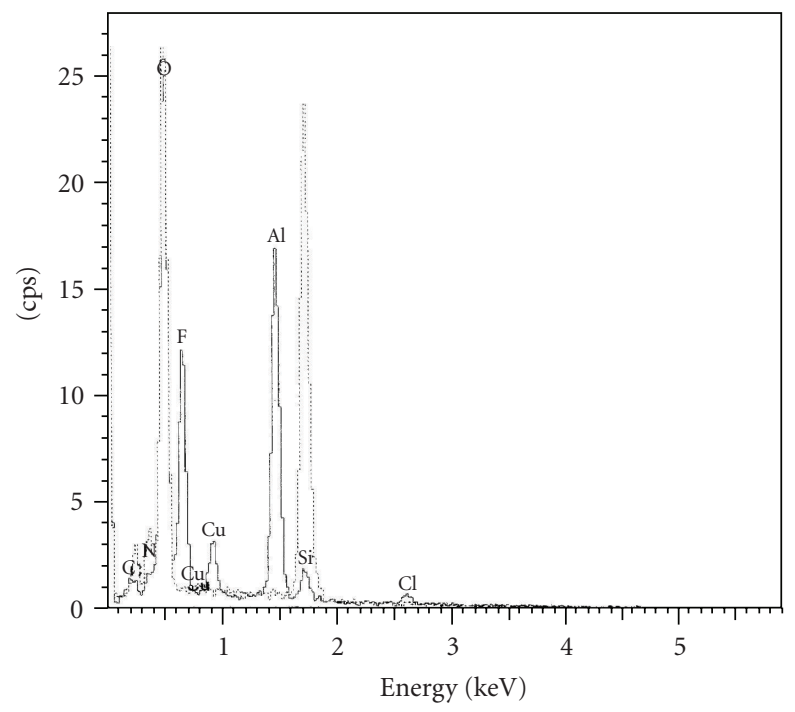

FIGURE 6: EDS for analyzing post metal etch residue. The residues contain $\mathrm{Al}, \mathrm{F}, \mathrm{Cu}, \mathrm{Si}$, and $\mathrm{Cl}$ atoms (solid line). The dashed line indicates TEOS background.

resulting in poor capability to remove the Al-containing residues. As expected in Figure 5(b), the extent of $\mathrm{Al}$ residue was decreased with decreasing $\mathrm{CHF}_{3}$ additive gas $(6 \mathrm{sccm})$ in the ME step; the ratio of $\mathrm{Al}$ residue to total defect adders was suppressed from $78 \%$ to $2.7 \%$. The reduction of the $\mathrm{CHF}_{3}$ flow rate in the ME step can effectively improve the post metal etch residue issue, but it will also result in less passivant on the metal sidewall to protect the Al bottom corner from the attack of $\mathrm{Cl}$ atoms. As shown in Figure 5(b), a slight bottom notching was observed on the Al corner. Therefore, there is a tradeoff between $\mathrm{Al}$ notching elimination and residue removal.

\subsection{Characterization and reduction of blocked etch metal island}

Blocked etch metal island is identified as a tangible yield risk in the oxide hard-mask-based $\mathrm{Al}$ etch manufacturing. SEM micrograph of metal island defect (see Figure 1(c)) revealed that it is a block of etched metal stack causing metal short 


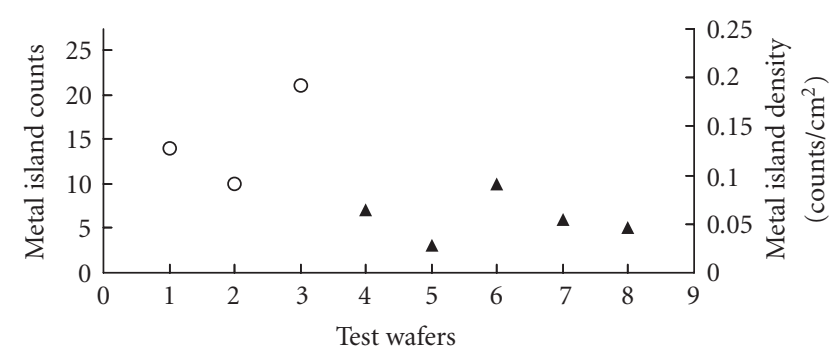

O Conventional metal etch w/o TiN surface pre treatment (metal island: 15 counts (average), Do $=0.14 \pm \sigma: 0.05$ counts $\left./ \mathrm{cm}^{2}\right)$

$\Delta$ Metal etch with TiN surface pre treatment (metal island: 6 counts (average), Do $=0.06 \pm \sigma: 0.02$ counts $/ \mathrm{cm}^{2}$ )

Figure 7: Trend of metal island defects versus metal etch recipe. The open circle symbol (o) presents metal etch using the conventional recipe without TiN surface pretreatment; the solid triangle symbol $(\boldsymbol{\Lambda})$ presents metal etch with TiN surface pretreatment prior to the TiN/Ti BT step.

between metal lines. The defect level on the wafers in Figure 7 showed 15 metal island adders in average and 21 metal island adders at maximum by implementing the starting metal etch baseline. There are several possibilities causing blocked metal etch such as the micromasking residue (e.g., $\mathrm{Ti}_{x} \mathrm{O}_{y} \mathrm{C}_{z}$ ) formed on the surface of TiN during the TEOS hard-mask etching with carbon fluoride chemistry [7] or the particulate flaking on the wafer from the etch chamber wall [8]. The possibility of blocked metal etch from the micromasking was investigated to lessen by adding a TiN surface pretreatment with $\mathrm{Ar} / \mathrm{CHF}_{3} / \mathrm{N}_{2}$ gas ratio (12/2/1) prior to the TiN/Ti BT step on metal etcher. As shown in Figure 7, the reduction of metal island adder was observed using the etch recipe with additional TiN surface pretreatment, inspecting six metal island adders in average and 10 metal island adders at maximum. The results reveal that the additional TiN surface pretreatment prior to the TiN/Ti BT step on metal etcher can reduce the impact of the micromasking on blocked metal etch significantly. Note that the particulate flaking from etcher wall was minimized by performing a preventive maintenance clean on the etch chamber in this study.

\section{CONCLUSIONS}

The etch defect investigations in this work are summarized as follows.

(I) Post hard-mask etch residue containing $\mathrm{C}$ atom is found strongly related to the hardening of photoresist after a $275^{\circ} \mathrm{C}$ ashing process. An in situ $\mathrm{O}_{2}$-based plasma ashing on RIE etcher is developed to prevent the photoresist hardening from the high-ashing temperature. The result reveals that such hardened polymeric residue on top of TEOS can be eliminated by optimizing in situ $\mathrm{O}_{2}$-based plasma ashing with $\mathrm{rf}$ power of $150 \mathrm{~W}$ for 12 seconds followed by EKC265 wet stripping.
(II) Post metal etch residue is demonstrated to compose of $\mathrm{Al}, \mathrm{F}, \mathrm{Cu}, \mathrm{Si}$, and $\mathrm{Cl}$ atoms. The data indicate that the residue is related to the attack of the $\mathrm{Al}$ $(0.5 \% \mathrm{Cu})$ sidewall by $\mathrm{Cl}$ atoms, and too much $\mathrm{CHF}_{3}$ addition in the $\mathrm{Al} \mathrm{ME}$ step can passivate the surface of $\mathrm{Al}$ resulting in poor capability to remove the Alcontaining residue. To lower the $\mathrm{CHF}_{3}$ additive gas from $15 \mathrm{sccm}$ to $6 \mathrm{sccm}$ in the $\mathrm{Al} \mathrm{ME}$ step will greatly improve the residue remaining issue.

(III) One possibility of blocked etch metal island creating is the fine micromasking formed on the opening of TiN during the hard-mask patterning. A TiN surface pretreatment with the $\mathrm{Ar} / \mathrm{CHF}_{3} / \mathrm{N}_{2}$ plasmas can be implemented prior to the TiN/Ti BT step to reduce the impact of the micromasking on blocked metal etch.

\section{REFERENCES}

[1] J. Yamamoto, T. Hsieh, T. Kondoh, et al., "A highly reliable aluminum interconnect etching process control for $0.13 \mu \mathrm{m}$ flash technology," in Proceedings of IEEE International Symposium on Semiconductor Manufacturing (ISSM '03), pp. 207-210, San Jose, Calif, USA, September-October 2003.

[2] M. Ronay, "Development of aluminum chemical mechanical planarization," Journal of the Electrochemical Society, vol. 148, no. 9, pp. G494-G499, 2001.

[3] S. Louwers, "Use of $\mathrm{W}$ damascene as true metall in a $130 \mathrm{~nm}$ flash technology," in Proceedings of the 1st International Conference on Memory Technology and Design (ICMTD '05), pp. 203-206, Giens, France, May 2005.

[4] C. T. Gabriel, R. Y. Kim, and D. C. Baker, "Integration of metal masking and etching for deep submicron patterning," Journal of Vacuum Science \& Technology A, vol. 18, no. 4, pp. 1420-1424, 2000.

[5] G. Stojakovic and X. J. Ning, "Reactive ion etch of $150 \mathrm{~nm} \mathrm{Al}$ lines for interconnections in dynamic random access memory," Journal of Vacuum Science \& Technology A, vol. 18, no. 4, pp. 1425-1430, 2000.

[6] P. Gillespie, I. Berry, and P. Sakthivel, "Wafer temperature control-a critical parameter for dry photoresist and residue removal," in Proceedings of the International Semiconductor Conference, Sinaia, Romania, October 1999.

[7] J. Song, J.-S. Kim, H. Seo, Y. Kim, and H. Jeon, "Removal of the metallorganic polymer residues formed at via holes," Journal of the Electrochemical Society, vol. 151, no. 5, pp. G323-G327, 2004.

[8] W. K. Au, J. Sappidi, D. Parks, et al., "Integration for reduction of sub-half-micron center yield fallout," in Proceedings of IEEE/SEMI Advanced Semiconductor Manufacturing Conference (ASMC '99), pp. 18-20, Boston, Mass, USA, September 1999. 